\title{
Peut-on réduire les inégalités dans le risque de cancer, sa prévention, son dépistage, l'accès aux traitements, le soutien des proches, la qualité de vie après sa guérison ?
}

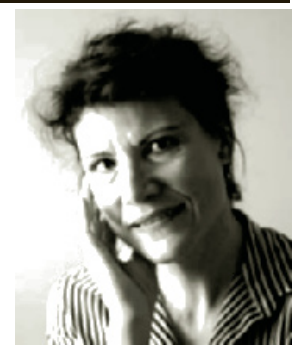

\author{
Can we reduce inequalities in cancer risk, prevention, screening, \\ access to treatment, support for the family and the quality of life after cure?
}

\author{
M.-F. Bacqué \\ (C) Springer-Verlag France 2010
}

Cette question résonne presque comme une boutade. Les sociétés libérales haussent les épaules en l'entendant, les idéalistes poursuivent le combat, les réalistes, dont nous sommes, observent, effarés, les multiples croisements de variables, puis mettent en chantier les premières réflexions. Ce $27^{\mathrm{e}}$ congrès de la Société française de psycho-oncologie, dont vous trouvez les principales communications dans le dernier numéro de l'année 2010 de Psycho-oncologie, est consacré aux inégalités d'origine psychique et aux conséquences psychiques des inégalités dans le cancer.

Les statistiques nous permettent un premier constat quantitatif et qui, pourtant, montre l'impact du cancer sur la population. Depuis 2009, 350000 nouveaux cas de cancer par an touchent une famille sur trois en France. Le cancer est aussi la première cause de mortalité avec environ 150000 morts qui lui sont directement liées.

Cependant, si entre 1980 et 2005, l'augmentation de nouveaux cas a dépassé $80 \%$, le taux de guérison a augmenté de $25 \%$. On rencontre donc de plus en plus de personnes atteintes, mais aussi guéries du cancer!

Ces chiffres expliquent pourquoi le cancer est un thème fréquemment cité, appelé sans doute à se populariser et à perdre l'image stigmatisante qui a contribué à le cacher pendant si longtemps.

Cependant, plus les méthodes des sciences humaines s'affinent et plus leurs résultats nous convainquent : nous formons une France inégalitaire, bien plus que celle, rêvée par les partisans de la Déclaration des droits de l'homme.

M.-F. Bacqué $(\bowtie)$

Université de Strasbourg,

département de psychologie,

12, rue Goethe, F-67000 Strasbourg, France

e-mail : mfbacque@club-internet.fr

\section{Géographie de la disparité de l'accès aux soins}

Les disparités les plus frappantes entre les populations proviennent surtout des lieux où elles vivent. La mortalité par cancer est la plus élevée dans les régions européennes les plus industrialisées (Belgique, Pays-Bas, Luxembourg, Danemark, Grande-Bretagne). Le sud de la France, l'Espagne, l'Italie et surtout la Grèce sont des régions à plus faible mortalité, de même que la Finlande et la Suède. Bien sûr, il existe des disparités régionales de l'incidence des cancers (en France, cette disparité est grande entre le Nord et le Sud), mais globalement, il se pourrait qu'au-delà de l'alcoolotabagisme et même de la pollution, l'industrialisation et sa production de bouleversements physique, chimique et biologique soient à l'origine de la première cause de mortalité en France.

Une des particularités des inégalités observées en France est l'inégalité géographique retrouvée de manière plus constante que les inégalités sociales - les deux sont le plus souvent liées. Dans l'étude menée par Dejardin et al., en 2006, sur plus de 80000 patients atteints d'un cancer digestif dans 12 départements français [2], le risque de ne pas survivre à un cancer colique augmente significativement de $10 \%$ ou de $14 \%$ pour les habitants à plus de $30 \mathrm{~km}$ ou de $50 \mathrm{~km}$ d'un centre de soins de référence. Les patients les plus éloignés voient leur survie péjorée de $25 \%$ lorsque le cancer est diagnostiqué à un stade tardif [1]. Plus les patients sont isolés socialement ou géographiquement, moins ils sont pris en charge de manière optimale. Le recours aux centres spécialisés est directement et significativement lié à sa proximité géographique du domicile du patient.

Rien de psychologique là-dedans soutiendra-t-on... Beaucoup de politique en revanche, lorsque l'on sait que la fermeture des petits hôpitaux régionaux risque d'augmenter la « fracture sociale de l'accès aux soins » entre les ruraux et les citadins ou proches des villes. 


\section{Disparité dans l'accès à la prévention}

L'éloignement des lieux de soins est un handicap, mais l'application des mesures de prévention en est un autre. Dans ce domaine, la pauvreté est aussi sûrement un obstacle que la compréhension d'un message pourtant ressassé par la télévision. Ce média "démocratique " par excellence ne peut pas rester le seul vecteur d'information ou de proposition de mode de vie. Nous comprenons que le croisement de plusieurs informations est bien plus efficace que des messages officiels, rapidement perdus dans le flot des nouvelles. L'accent mis sur la prévention doit donc être renforcé, mais de façon plus précise, lorsqu'il est ciblé sur des populations en situation de précarité. Et il ne s'agit pas de messages Internet ou de SMS, mais bien de mobiliser les femmes et les hommes à rencontrer, écouter, puis s'adresser à la bonne personne. Ceux qui errent sans domicile, les exilés qui cherchent un pays d'accueil, les toxicomanes, les malades mentaux, les chômeurs, les adolescents en situation de crise, les personnes âgées désocialisées, tous ont besoin de cette proximité pour comprendre. Ils ont aussi d'autres priorités comme trouver un toit, manger...

Rappelons que certains pays comme le Mexique ou le Bangladesh ont proposé des programmes pilotes qui paient les personnes les plus fragiles pour appliquer les mesures de prévention et limiter ainsi les risques d'hospitalisation ou de traitement bien plus coûteux par la suite.

\section{Désocialisation et dépression}

Et comme un malheur ne vient jamais seul, il y a encore croisement entre toutes ces composantes d'une vie. La désocialisation renvoie à la solitude, la solitude à l'absence de pensée au lendemain, la perte des projets à la dépression...

La dépression est associée à une augmentation de l'incidence du cancer. Cela ne veut pas dire qu'il existe un lien de causalité direct entre dépression et cancer, mais on pourrait plutôt parler de comorbidité. Les conséquences de la dépression, en termes d'augmentation de la consommation alcoolotabagique, de la sédentarité excessive ou d'une alimentation dégradée sont bien connues. Celles qui portent sur le retard au dépistage ou au début des traitements pourraient être maintenant mieux prises en compte par les organisations de santé publique comme par les personnels des hôpitaux psychiatriques (voir le $\mathrm{n}^{\circ}{ }^{\circ} 1,2010$ de Psychooncologie). Si la dépression est difficile à repérer et à traiter dans la population générale, elle l'est encore plus dans les services d'oncologie où sa symptomatologie est parfois confondue avec les symptômes dépressifs entrainés par le cancer et son diagnostic.

La dépression est aussi un symptôme transversal retrouvé dans d'autres maladies psychiatriques. Nous avons large- ment insisté sur les effets délétères de la maladie mentale sur le dépistage des cancers. Le soutien des psychotiques, et des schizophrènes en particulier, devrait consacrer toujours plus de place à leur santé somatique.

\section{La rareté implique souvent l'inégalité}

Les cancers rares provoquent souvent la sidération. Ils sont aussi souvent oubliés. Les cancers d'origine génétique, bien que ne représentant qu'environ $8 \%$ parmi les cancers, requièrent une spécificité dans leur accompagnement. Les questions d'anticipation et de révélation y sont particulières et sont aujourd'hui mieux comprises dans la double approche systématique, psychologique et somatique. Ce qui semble évident dans cette consultation, où les aspects psychologiques (la culpabilité, l'angoisse de transmettre la maladie, les décisions douloureuses sans certitude de développer la maladie) sont pris en compte, devrait être élargi à tous les cancers.

Un avenir en berne dès le diagnostic génétique d'un cancer peut briser tout espoir de vie et d'adaptation, les équipes pluridisciplinaires l'ont bien compris et forment un modèle qui pourrait être conjugué dans tous les services d'oncologie.

\section{Élargir l'approche psychosociale}

Ainsi, il apparaît que les médecins, les assistants de service social et les professionnels de la psyché peuvent limiter les inégalités, une fois le cancer installé, en étant présentés clairement dans les services et en mobilisant leurs efforts pour limiter les conséquences du cancer.

Pour les causes environnementales, les maladies professionnelles, les campagnes de prévention et de dépistage, les pouvoirs publics dirigent leurs actions de façon souvent trop lente et parfois ambiguë, comme dans le cas des maladies professionnelles (statut accordé à la portion congrue) ou dans le cas des conséquences environnementales de l'industrialisation d'une région. Notre société a une vocation d'expression politique. Elle ne peut pas seulement se contenter de constater les inégalités. Elle soulève les objections qui apparaissent dans la faiblesse du soutien des plus pauvres, de ceux qui vivent dans la rue ou de ceux qui passent dans notre pays à la recherche d'une nationalité.

\section{Quelques bonnes nouvelles concernant la revue Psycho-oncologie}

Pour finir sur des notes plus optimistes, je voudrais souligner combien Psycho-oncologie a progressé depuis sa première parution en 2002. Le congrès de la SFPO à Caen, en octobre 
2001, avait donné l'occasion d'une réflexion stimulante sur la création d'une revue francophone, s'imposant dans le domaine de la psycho-oncologie. Presque dix années plus tard, Psycho-oncologie est bien présente, quatre fois par an, apportant une contribution non négligeable aux soins, mais aussi à l'approche transdisciplinaire du cancer. Elle a su tenir son pari d'ouverture aux sciences humaines et de partenariat avec la médecine. Psycho-oncologie a obtenu un impact factor auprès de l'ISI Web of Knowledge. Cela signifie qu'elle est une revue qualifiante au même titre que les plus grandes revues anglo-saxonnes. Même si l'impact factor est relativement faible du fait de la diminution d'influence de la langue française, il est tout de même de 0,253 sur les cinq dernières années. Les articles de Psychooncologie doivent être cités le plus souvent possible dans d'autres revues que Psycho-oncologie, cependant c'est une discipline à prendre pour tous nos lecteurs et auteurs que de diffuser nos références dans leurs articles.

Je rappelle donc que nous figurons dans de nombreuses bases de données internationales Science Citation Index Expanded (SciSearch ${ }^{\circledR}$ ), Journal Citation Reports/Science Edition, PsycInfo, Google Scholar, Scopus et nationales comme Pascal (INIST-CNRS).

Psycho-oncologie doit également publier des auteurs étrangers en anglais, afin d'être lue par un public international, ce qui explique nos efforts de « recrutement » lors des congrès de l'IPOS ou de l'APOS (International et American Psycho-oncology Societies). Une revue française avec quelques articles en anglais a plus de chances d'être lue à l'étranger et d'être citée dans les autres journaux. Considérons cet appel comme une proposition égalitariste dans un monde non francophone, mais où l'ensemble des scientifiques partagent au moins une langue commune...

\section{Références}

1. Dejardin O, Bouvier AM, Faivre J, et al (2008) Access to care, socioeconomic deprivation and colon cancer survival. Aliment Pharmacol Ther 27(10):940-9

2. Dejardin O, Remontet L, Bouvier AM, et al (2006) Socioeconomic and geographic determinants of survival of patients with digestive cancer in France. Br J Cancer 95(7):944-9

\section{Comité d'experts}

Au nom du comité de rédaction, je remercie les personnes qui ont accepté d'expertiser à l'aveugle les articles proposés à Psycho-Oncologie : Pr. François Baillet - Dr. Dana Castro Dr. Lionel Dany - Pr. Marie Santiago Delefosse - Pr. Nicole Delvaux - Dr. Martine Derzelle - Pr. Pascal-Henri Keller Pr. Bernard Hoerni - Pr. Bernard Lebeau - Pr. Marguerite Lederbergh - Dr. Daniel Lemler - Pr. Serge Lesourd - Pr. Eva Louvet - Dr. Daniel Oppenheim - Pr. Jean-Louis Pédinielli Dr. Christine Raynaert - Pr. Josée Savard - Dr. Anne Thevenot Pr. Claude de Tychey. Merci également à tous les auteurs. 